\title{
Full-in-House Method (FinHM) for SARS-COV-2 Automated Viral RNA Extraction, Followed by in-House 'Primer-Probe' Based RT-qPCR Detection; Low Cost Mass Testing
}

\author{
Khaldoun Al-Romaih ${ }^{1}$, Ibtihaj Alsharif ${ }^{1}$, Razan Bakheet ${ }^{1}$, Lina Mahmoud ${ }^{1}$, Najla Alharbi ${ }^{1}$, \\ Sara Bin Judia ${ }^{1}$, Layla Alharbi ${ }^{1}$, Abdulaziz Alzayed ${ }^{2}$, Amjad Jabaan ${ }^{2}$, Jawahar Alotaibi ${ }^{1}$, \\ Alaiya Ayodele ${ }^{1}$, Zakiya Shinwari ${ }^{1}$, Hani Alhadrami ${ }^{3}$, Ahmed Albarrag ${ }^{4}$, Esam Azhar ${ }^{3}$, \\ Haya Al-Saud ${ }^{2}$, Tahani Alrahbini, ${ }^{1,5}$, Maha Al-Mozaini ${ }^{1, *}$ \\ ${ }^{1}$ King Faisal Specialist Hospital and Research Centre, Riyadh, Kingdom of Saudi Arabia \\ ${ }^{2}$ King Abdulaziz City for Science and Technology, Riyadh, Kingdom of Saudi Arabia \\ ${ }^{3}$ King Abdulaziz University Hospital, Jeddah, Kingdom of Saudi Arabia \\ ${ }^{4}$ Saudi Center for Disease Prevention and Control, Riyadh, Kingdom of Saudi Arabia \\ ${ }^{5}$ Riyadh Elm University, Riyadh, Kingdome of Saudi Arabia
}

Email address:

mmozaini@kfshrc.edu.sa (M. Al-Mozaini)

${ }^{*}$ Corresponding author

To cite this article:

Khaldoun Al-Romaih, Ibtihaj Alsharif, Razan Bakheet, Lina Mahmoud, Najla Alharbi, Sara Bin Judia, Layla Alharbi, Abdulaziz Alzayed, Amjad Jabaan, Jawahar Alotaibi, Alaiya Ayodele, Zakiya Shinwari, Hani Alhadrami, Ahmed Albarrag, Esam Azhar, Haya Al-Saud, Tahani Alrahbini, Maha Al-Mozaini. Full-in-House Method (FinHM) for SARS-COV-2 Automated Viral RNA Extraction, Followed by in-House 'Primer-Probe' Based RT-qPCR Detection; Low Cost Mass Testing. American Journal of Internal Medicine. Vol. 9, No. 1, 2021, pp. 1-10. doi: 10.11648/j.ajim.20210901.11

Received: December 7, 2020; Accepted: December 21, 2020; Published: January 4, 2021

\begin{abstract}
Background: Sever acute respiratory syndrome Coronavirus-2 (SARS-COV-2) spread prompted mass testing. The main method for testing is by any FDA approved kits for RNA extraction followed by One-Step RT-qPCR based on primerprobe assays. Yet, the high demand for these kits created a global bottleneck in the testing capacity. Methods: We developed a Full-In-House Method (FinHM) suitable for automated viral RNA extraction using full in-house solutions utilizing the MagMax $^{\mathrm{TM}}$ beads followed by an In-House RT-qPCR based on the CDC/WHO recommended 'primer-probe' assay targeting the following genes; E, RdRp2, and RdRp4. FinHM was validated by an FDA approved kit that targets S, N, and ORF1b genes made by Thermo Fisher Scientific (TF). Results: The sensitivity and specificity of the automated RNA extraction were evaluated on serial dilutions of in-laboratory propagated SARS-COV-2 with a successful detection down to 46 copies in both assays $(\mathrm{P}>0.05)$. Moreover, automated FinHM was successful in extraction of SARS-COV-2 RNA in 266 clinical samples, in which the test results replicated the FDA approved test results ( $>99 \%$ similarity, P $>0.05)$. The In-House RT-qPCR assay had low limit of detection (5 RNA templates), with significant negative correlation between the Ct values and RNA titrations as shown by Pearson correlation (-0.8, -0.8 and -0.7 for E, RdRp2 and RdRp4, respectively). Finally, FinHM was also successful in extraction of SARS-COV-2-spiked plasma and patient plasma samples. Conclusion: We report a reliable, reproducible, specific, sensitive and low-cost platform for automated RNA extraction and detection from SARS-COV-2 and other viruses which is suitable for clinical and mass testing.
\end{abstract}

Keywords: SARS-COV-2, High Throughput Testing, One Step RT-qPCR, Primer-Probe, Diagnostic Testing

\section{Background}

Communities, worldwide, keep facing emerging pathogens, some of which cause pandemic situations. In less than 2 decades, there was spread of viral infections caused by viruses belonging to Coronaviridae (CoVs) 
family of viruses, namely coronaviruses. Coronaviruses are RNA viruses that infect birds, mammals, and humans and it can affect different systems including the respiratory system [1]. At least 7 coronaviruses are known to infect humans and cause diseases of variable severities. From amongst these 7 coronaviruses are the Severe Acute Respiratory Syndrome Coronavirus-1 (SARS-CoV-1; 2002 - 2003 outbreak) and the Middle East Respiratory Syndrome Coronavirus (MERS-CoV; 2012 outbreak) [1], and the most recently discovered and ongoing pandemic source; Severe Acute Respiratory Syndrome Coronavirus-2 (SARS-CoV-2; 2019 - to date outbreak) [2]. SARS-CoV-2 spread prompted world-wide ongoing mass and rapid diagnostic testing for several millions of people per day. The main method used to test subjects for SARS-CoV-2 infection is based on Reverse Transcription-quantitative Polymerase Chain Reaction (RT-qPCR) which is performed after RNA extraction [3, 4]. The Centre for Disease Prevention and Control (SCDC) and the World Health Organization (WHO) has published and recommended six different sets of primer and probe sets for different genes to develop an in-house SARS-CoV-2 assays. These different sets of primers and probes have been tested for both their sensitivity and specificity for SARS-CoV-2. These assays also have minimal cross reactivity with other circulating strains of coronaviruses and recommended the use of 40 cycle as a cutoff point and threshold for SARS-COV-2 positivity [5].

There are several kits that employ these approaches but due to the high demand in the last few months resulted in the shortage of these kits and prompted the use of alternative resources. To counter this shortage, we developed an automated method for viral RNA extraction using an in-house solutions and reagents utilizing only the commercially available MagMax $^{\mathrm{TM}}$ beads for RNA extraction followed by a PCR assay based on primers and probes recommended by CDC and WHO for SARS-COV-2 testing. The in-house solutions and reagents are basic, affordable, and used commonly in any laboratories lowering the cost to around $\$ 0.80$ per sample for the extraction process. This approach is also simple to perform and suitable for mass production and automation which potentiates mass testing. In addition, the detection of the extracted RNA using the CDC/WHO recommended primerprobe assay was cross validated by an FDA approved kit on SARS-COV-2 propagated virus in the laboratory as well as to patient nasopharyngeal samples and plasma samples. In conclusion, our approach provides an alternative protocol to counter the shortage in SARS-COV-2 diagnostic testing with low-cost mass testing.

\section{Methods}

\subsection{Sample Collection and Ethical Considerations}

The study was conducted according to the Declaration of Helsinki. This study was approved by the institutional review board at King Faisal Specialist Hospital and Research Centre, Riyadh (KFSHRC-Riyadh) IRB, (RAC Approval \# 2200031) the board also granted a waiver for obtaining informed consent owing to the use of de-identified archived samples for this study.

Two hundred and sixty six positive samples for SARSCOV-2 $(\mathrm{N}=266)$ and eight negative samples for SARS-COV$2(\mathrm{~N}=8)$ were included used controls. These samples are archived and de-identified and were collected from each patient using a nasopharyngeal swab for diagnostic purposes by the Saudi Center for Disease Prevention and Control (SCDC) then the left-over of these samples were deidentified, coded and used for this study to validate our FinHM platform. In addition sixteen plasma de-identified samples ( $\mathrm{N}=16$; also leftover from diagnostic material) were shared for SARS-COV-2 viral shedding by the Department Pathology and Laboratory Medicine at KFSHRC-Riyadh (DPLM-Riyadh).

\subsection{Propagating the SARS-COV-2 Virus}

The Vero E6 cells (ATCC ${ }^{\circledR}$ CRL-1586TM) were cultured at $37^{\circ} \mathrm{C}$ in $5 \% \mathrm{CO} 2$. The local SARS-CoV-2 clinical isolate (SARS-CoV-2/human/SAU/85791C/2020) (Gene accession number MT630432.1) was propagated and titrated using Median Tissue Culture Infectious Dose method (TCID50). SARS-COV-2 was propagated and the virus isolates were heat inactivated, diluted as indicated in the results section, and used for the assays in biosafety level-3 facility.

\subsection{Full In-house Method (FinHM) for Beads-based Viral RNA Extraction}

Robotic systems Hamilton's Microlab STAR liquid handling system or the Thermo Fisher Scientific's KingFisher Flex System was used for automated RNA extraction. Initially $200 \mu \mathrm{l}$ of viral transport medium and plasma from a donor, negative for SARS-COV-2, were spiked with propagated SARS-COV-2 or MS2 (RNA bacteriophage) with the following titrations: MS2 $(10 \mu \mathrm{l}$ to $0.625 \mu \mathrm{l})$, SARS-COV-2 in viral transport media (3000 down to $<10$ viral copies) and SARS-COV-2 virus spiked in plasma (500 down to $<10$ viral copies). We then combined these titrated samples with $100 \mu \mathrm{l}$ of Guanidinium Thiocyanate (GITC) lysis buffer ( $\mathrm{pH}$ 6.5) (Lysis Buffer made of $6 \mathrm{M}$ GITC, $50 \mathrm{mM}$ Tris-Hcl, $20 \mathrm{mM}$ EDTA and $2 \%$ Tween 20 ). Followed by the addition of 20 $\mu 1$ of MagMax $^{\mathrm{TM}}$ magnetic beads (Thermo Fisher, A42362) to each sample with lysis buffer, subsequent washes was done by adding $100 \%$ Ethanol $(270 \mu \mathrm{l})$, followed by mixing 4-6 times with pipetting and 1 minutes incubation at room temperature to allow the RNA to bind to the beads. For the automation process the beads and ethanol are premixed and added to the lysis mix to improve the workflow. The Plate were then placed on the magnetic dock for 10 minutes, then the supernatant is aspirated while the beads are bounded to the bottom of plate via the magnetic dock. Subsequent washes were then done again with $100 \%$ Ethanol $(150 \mu \mathrm{l})$ 
as a first wash, followed by two washes with $75 \%$ Ethanol $(150 \mu 1)$. For the automation process we performed the washing steps away from the magnetic dock followed by a 5 minutes of positioning the plate on the magnetic dock for a full bead attachment at the bottom of the plate before the aspiration step of supernatant. After the final wash, the beads are left on the magnetic dock to remove all the excess Ethanol (up to 5 minutes). The plate is then removed away from the magnetic dock and RNA-bound beads are resuspended in RNAse free water $(40 \mu \mathrm{l})$ and allowed to elute for $2 \mathrm{~min}$. Finally the plate was placed on the magnetic dock for 2 minutes and the eluted RNA is aspirated and transferred to a PCR plate for RT-qPCR step. We added 1 $\mu l$ of RNaseOut (Invitrogen, USA) to inhibit RNases and to protect the RNA from degradation.

\subsection{Designing the Primers and Probes for the $S A R S-C O V-2 R T-q P C R$}

Primers and probes for our In-House assay were designed based on WHO and CDC recommendations for patient testing and diagnosis. They were adapted by Charité Institute of Virology, pasture institute in Paris [6, 7]. The assay specifically targets the $\mathrm{E}$ gene (single-tube) for beta coronaviruses while the other specific genes RdRp2, and RdRp4 (duplexing) (Table 1). For our in-house PCR assay we designed all these primers with different dyes to achieve a single tube assay (multiplexing). These primers and probes were made by oligo-synthesis followed by HPLC-grade purification for maximum primer-probe purity (Metabion, Germany).

Table 1. Primers and probes for the in-house SARS-CoV-2 RT-qPCR diagnostic assay.

\begin{tabular}{lll}
\hline Target & Primer and probes & Sequence* \\
\hline \multirow{2}{*}{ E } & E_Sarbeco_Reverse & ACA GGT ACG TTA ATA GTT AAT AGC GT \\
& E_Sarbeco_Forward & ATA TTG CAG CAG TAC GCA CAC A \\
& E_Sarbeco_Probe & ACA CTA GCC ATC CTT ACT GCG CTT CG [5'] TAMRA [3'] BHQ-1 \\
RdRp 2 & RdRp/nCoV_IP2-Forward & ATG AGC TTA GTC CTG TTG \\
& RdRp/nCoV_IP2-Reverse & CTC CCT TTG TTG TGT TGT \\
& RdRp/nCoV_IP2-Probe & AGA TGT CTT GTG CTG CCG GTA [5'] HEX [3'] BHQ-1 \\
RdRp/nCoV_IP4-Forward 4 & GGT AAC TGG TAT GAT TTC G \\
& RdRp/nCoV_IP4-Reverse & CTG GTC AAG GTT AAT ATA GG \\
& RdRp/nCoV_IP4-Probe & TCA TAC AAA CCA CGC CAG G [5'] FAM [3'] BHQ-1 \\
\hline
\end{tabular}

*Adapted from Charité Institute of Virology [7].

\subsection{In-house Multiplexing One Step RT-qPCR Assay}

For the ThermoFisher assay: $10 \mu \mathrm{l}$ of the extracted RNA (The final eluted material obtained at the end of the automated viral RNA extraction) was used as input material for the multiplex single-step PCR for 3 genes using the Thermo Fisher kit COVID PCR TaqPath ${ }^{\circledR}$ kit (ABI: A48102) as per manufacturer protocol (annealing at $58^{\circ} \mathrm{C}$, TaqPath ${ }^{\circledR}$ One-Step qRT-QPCR system on the ABI 7500 real time cycler for a total of $25 \mu \mathrm{l}$ per reaction). The In-House primer-probe assay was done for each patient in single tube by a single-step real-time qPCR that specifically targets the E gene, RdRp2 and RdRP4 genes (multiplexing). The run was performed using these conditions: the reaction mixed contains the primers and probes $(1 \mu \mathrm{l}$ each $/ 10 \mu \mathrm{M}), 1 \mu \mathrm{l}$ SuperScript $^{\mathrm{TM}}$ III Platinum ${ }^{\mathrm{TM}}$ OneStep qRT-PCR Kit (Invitrogen cat: 11732088) and $1 \mu 1$ of DNase/RNase-free water with the addition of $50 \mathrm{mM} \mathrm{MgSO} 4$ $(0.4 \mu \mathrm{l})$ to enhance the catalytic activity of the enzyme, especially in multiplexing protocol. The PCR cycle was performed as follows: first the RNA conversion to cDNA at $55^{\circ} \mathrm{C}$ for 20 minutes for one cycle, followed by a denaturation step at $95^{\circ} \mathrm{C}$ for 3 minutes; then amplification for 40 acquisition cycles at $58^{\circ} \mathrm{C}$ for 30 seconds, and finally a cooling step at $40^{\circ} \mathrm{C}$ for 30 seconds.

In each PCR assay we have analyzed the samples with different sets of controls in each run, as follows; (1) a NonTemplet control (NTC), (2) Titrated Positive control which is SARS-COV-2 RNA template from 50,000 copies to $<10$ copies for limit of detection to establish a quantitative viral load measurements. (3) We also included a negative control for the test, which is an RNA template for SARS-COV-1 Frankfurt strain, for specificity. The SARS-COV-1 and SARSCOV-2 RNA template was obtained from the European Archives of Virology, Ref-SKU: 026N-03889, 1e4 copies/ $\mu$ l).

\section{Results}

\subsection{FinHM Successfully Isolated MS2 and the Propagated SARS-COV-2, Spiked into a Medium for Subsequent Detection Using TaqPath ${ }^{\circledR}$ One-Step RT-qPCR}

To validate the capacity of our FinHM platform in extracting the viral RNA we spiked a growth medium (with $5 \%$ BSA) with an internal control MS2 (Single-strand RNA), a bacteriophage provided in the Applied Biosystems (ABI) assay (Applied Biosystems, catalogue number A48102) and with propagated whole virus SARS-COV-2 at different titrations. The stepwise dilution of the internal control of MS2 was conducted (from $10 \mu \mathrm{l}$ to $0.625 \mu \mathrm{l}$ ) and SARS-COV-2 (3000 copies down to $<10$ copies) and were spiked and then conducted a series of automated RNA extractions using our FinHM extraction platform. A standard one-step RT-qPCR was used to detect bacteriophage MS2 RNA and SARS-COV-2 genes after the automation process (for each spiking experiments). The purified RNA samples were tested by RT-qPCR assay by using the Thermo Fisher Scientific COVID PCR TaqPath (Applied Biosystems, catalogue number A48102) targeting three different viral genes; S, N, and ORF1b genes. As 
shown in table 2 and 3 the automated extraction using FinHM isolated sufficient RNA for detection by RT-qPCR. The cycle threshold $(\mathrm{Ct})$ at which each RNA product was detected are from $\mathrm{Ct}$ of 22.53 to 39.49 for MS2 RNA, Ct of 18.23 to 27.44 for $\mathrm{S}$ gene, $\mathrm{Ct}$ of 18.22 to 25.09 for ORF1b gene and $\mathrm{Ct}$ of 15.18 to 26.85 for $\mathrm{N}$ gene. Our in-house assay $\mathrm{Ct}$ values for each RNA product was also detected and reported from $\mathrm{Ct}$ of 22.94 to 29.04 for $\mathrm{E}$ gene, $\mathrm{Ct}$ of 23.27 to 29.55 for RdRp2 gene and Ct of 24.38 to 29.44 for RdRp4 gene. Our assay showed specificity with reported limit of detection of 46 copies of SARS-COV-2 in the extraction step. Amplification curves for the indicated targets produced by RT-qPCR after automated FinHM in extracting of viral RNA are shown in Figure 1.

Table 2. MS2 titrations from 10 ul to 0.125 with the corresponding CT value of PCR Detection of after FinHM extraction process.

\begin{tabular}{ll}
\hline Titrations & Ct \\
\hline MS2 10ul & 20.53 \\
MS2 5ul & 22.05 \\
MS2 2.5ul & 25.87 \\
MS2 1.25ul & 25.69 \\
MS2 0.625 & 30.62 \\
MS2 0.312 ul & 39.61 \\
MS2 $0.156 u l$ & 36.49 \\
\hline
\end{tabular}
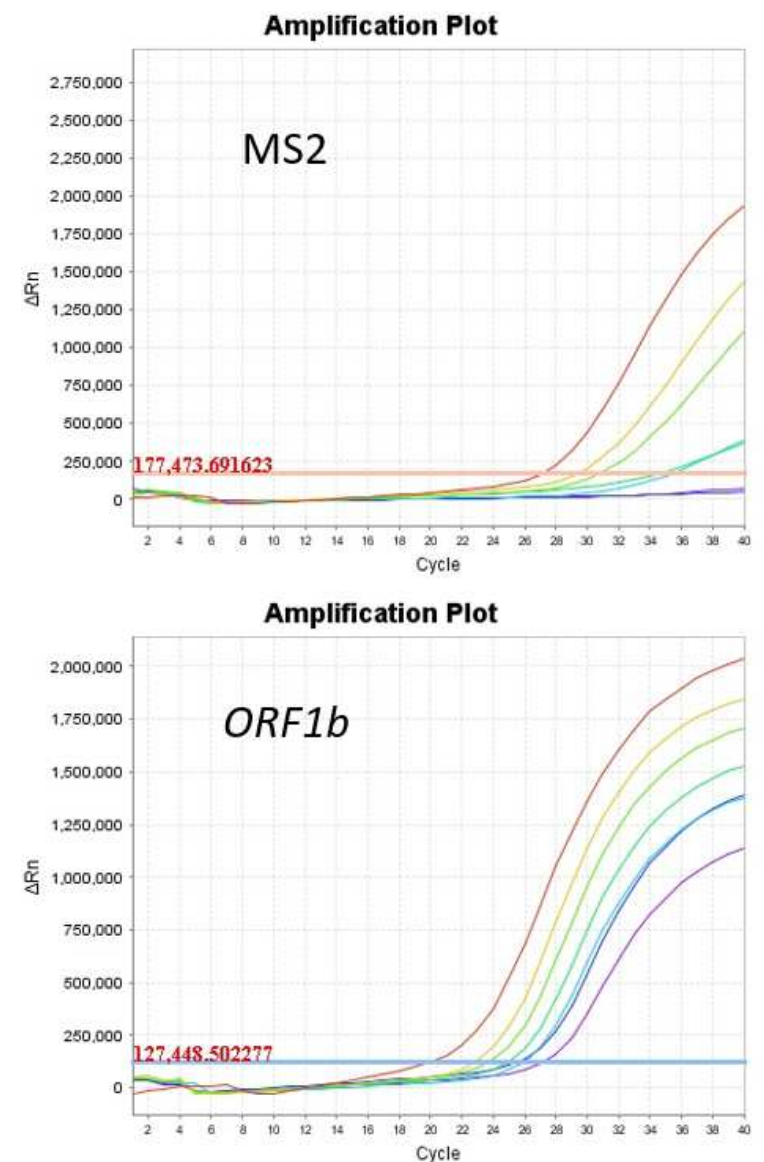

Amplification Plot

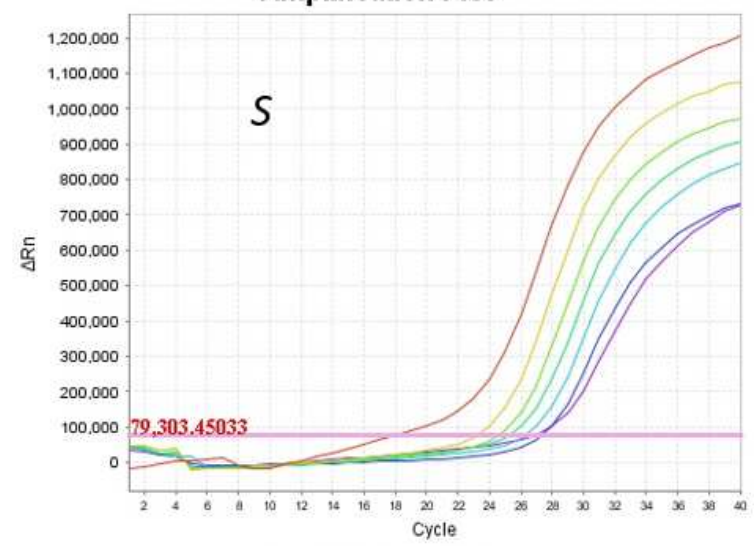

Amplification Plot

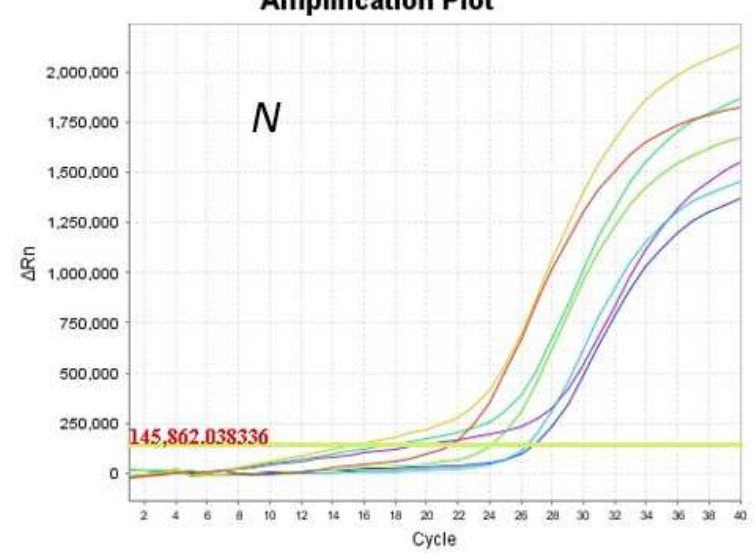

Figure 1. Detection of titrated SARS-COV-2 virus using ThermoFisher kit COVID PCR TaqPath assay after FinHM viral RNA extraction. Amplification curves for the indicated targets produced by RT-qPCR after automated FinHM in extracting of viral RNA. MS2, internal RNA extraction control, (bacteriophage provided in the Applied Biosystems assay, Applied Biosystems, catalogue number A48102) and SARS-COV-2 virus propagated in the laboratory at different titrations. A standard one-step RT-qPCR was used to detect bacteriophage MS2 RNA after the automated RNA extraction (spiking experiments). Yaxis is $\triangle R n$ and $X$ axis is cycle thresh-hold. MS2 is a positive-sense single-stranded RNA bacteriophage.

\subsection{FinHM Successfully Isolated the Propagated SARS-COV-2 That Was Spiked into Medium for Subsequent Detection Using Our In-house One-Step $R T-q P C R$}

To further validate the capacity of our developed automated FinHM to extract RNA from the propagated SARS-COV-2 we have designed primers and probes that targets three different SARS-COV-2 genes; E, RdRp2, RdRp4 and N. A single-step RT-qPCR was used to detect the propagated SARS-COV-2 virus after the automated RNA extraction in a series of dilutions (3000 down to $<10$ copies) using FinHM (Figure 2 and Table 3). Our in-house assay $\mathrm{Ct}$ values for each RNA product was detected and reported from Ct of 22.94 to 29.04 for for E gene, Ct of 23.27 to 29.55 for RdRp2 gene and Ct of 24.38 to 29.44 for RdRp4 gene. Our assay showed high sensitivity with reported limit of detection down to 46 copies of SARS-COV-2 in the extraction step as well as high specificity seen by the detection of at least one of the RdRp genes. The automated extraction platform using FinHM was capable to isolate sufficient RNA for the 
detection by RT-qPCR to the lowest titration (46 copies) of SARS-COV-2 (Ct of 29.04 for E gene, and Ct of 29.55 for RdRp2 gene and Ct of 29.44 for RdRp4 gene). Amplification curves for the indicated targets (E, RdRp2 and RdRp4) produced by RT-qPCR after automated FinHM in extracting of viral RNA from propagated SARS-COV-2 viruses at different titrations are shown in Figure 2. Furthermore, the detection capacity of ThermoFisher assay was similar to that of our In-House CDC recommended assay with no significant difference across the titrations $(\mathrm{P}>0.05)$. Thus, these results indicate that our automated FinHM extraction platform is robust and effective in the isolation and detection of SARS-COV-2 viral RNA. In addition, our in-house PCR assay is as efficient as the FDA approved assay.
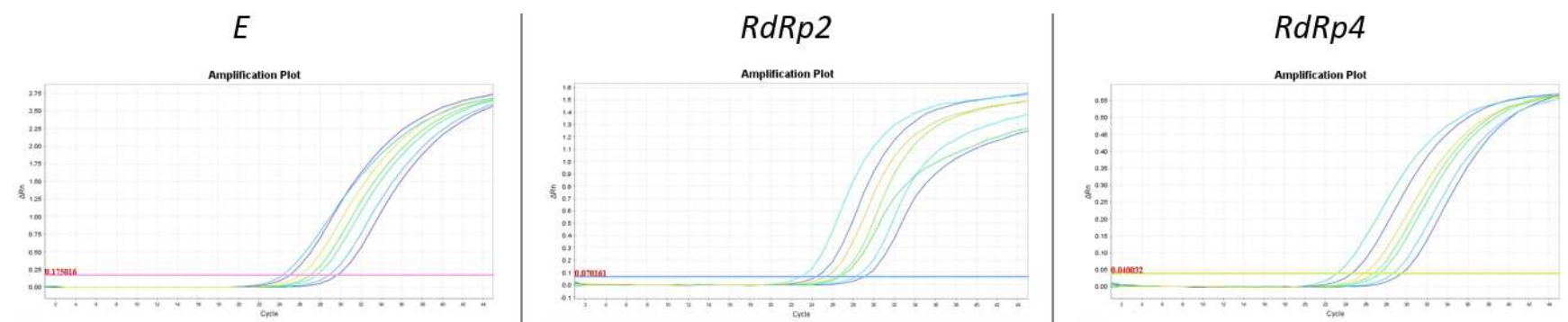

Figure 2. Detection of titrated SARS-COV-2 virus using in-house assay after FinHM viral RNA extraction. Amplification curves for the indicated targets (E, RdRp2 and RdRp4) produced by RT-qPCR after automated FinHM in extracting of viral RNA from propagated SARS-COV-2 viruses at different titrations. A standard one-step RT-qPCR was used to detect SARS-COV-2 genes after the automated RNA extraction. Yaxis is $\triangle R n$ and X axis is cycle thresh-hold.

Table 3. The limit of detection of titrated SARS-COV-2 virus after FinHM automated viral RNA extraction process using both ThermoFisher assay vs in house PCR assay.

\begin{tabular}{|c|c|c|c|c|c|c|c|c|c|c|c|}
\hline \multirow{2}{*}{$\begin{array}{l}\text { SARS-COV-2 } \\
\text { (Copy number) }\end{array}$} & \multicolumn{5}{|c|}{ Ct (ThermoFisher) } & \multicolumn{5}{|c|}{ Ct (In-House) } & \multirow{2}{*}{ P Value (TF vs SCDC) } \\
\hline & $S$ & ORF1b & $N$ & AVG & STDEV & $E$ & $R d R p 2$ & $R d R p 4$ & AVG & STDEV & \\
\hline 3000 & 18.23 & 18.02 & 21.43 & 19.22 & 1.91 & 22.94 & 23.27 & 24.38 & 23.53 & 0.76 & 0.02 \\
\hline 1500 & 23.36 & 21.81 & 15.18 & 20.11 & 4.35 & 24.45 & 24.69 & 25.04 & 24.73 & 0.30 & 0.23 \\
\hline 375 & 25.37 & 23.90 & 17.51 & 22.26 & 4.18 & 26.44 & 26.79 & 27.00 & 26.74 & 0.28 & 0.22 \\
\hline 187.5 & 26.39 & 25.02 & 26.31 & 25.91 & 0.77 & 26.82 & 27.36 & 27.64 & 27.27 & 0.42 & 0.13 \\
\hline 93.75 & 27.44 & 23.82 & 26.85 & 26.03 & 1.94 & 28.27 & 28.69 & 28.89 & 28.62 & 0.32 & 0.16 \\
\hline$<10$ & $* U D$ & UD & UD & 0 & 0 & 0 & UD & UD & UD & 0 & 0 \\
\hline
\end{tabular}

*UD is Undetermined.

ThermoFisher

In House

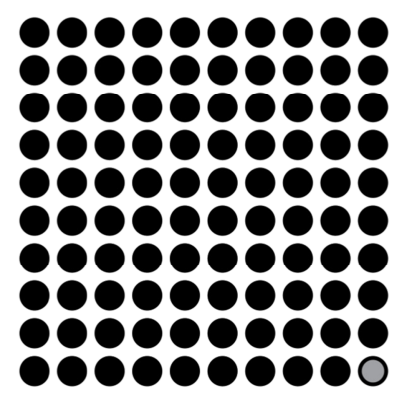

98.87\% Positive

$1.13 \%$ Negative

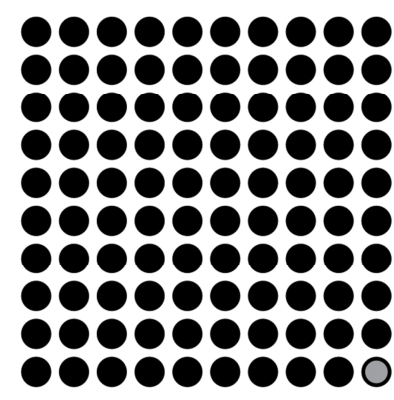

98.87\% Positive $1.13 \%$ Negative

\section{Total=266}

Figure 3. Comparison of the Thermofisher and the inhouse method results. The total number of positive samples supplied from CDC was 266. Each sample was analyzed via the two methods. For the Thermo fisher method, 3 samples were negative (1.13\%) while 263 were all positive (98.87\%), the inhouse method reported similar results. Chi-square and fishers exact analysis showed no significant difference between the outcomes of both methods with a P-value (>0.999).

\subsection{Automated FinHM Successfully Isolates SARS-COV-2 Viral RNA Form Patient Samples for Subsequent Detection Using the In-house Primer-probe PCR Assay}

We then moved to testing the capacity of the FinHM extraction platform and to validate it on SARS-COV-2 nasopharyngeal swabs obtained from patient samples $(\mathrm{N}=266)$ and eight known negative samples for SARA-COV$2(\mathrm{~N}=8)$. The sample were split into two parts and processed in parallel by 1) the automated Full in-House extraction platform followed by our standard single-step RT-qPCR to detect SARS-COV-2 RNA using our in-house PCR assay that targets the three different viral genes; E, RdRp2, and RdRp4 genes. 2) the samples were also analyzed and validated with the commercial kit "Thermo Fisher Kit's extraction and PCR assay that targets three SARS-COV-2 three other genes (ORF1a, N and S). For the Thermofisher method, 3 samples were negative $(1.13 \%)$ while 263 were all positive $(98.87 \%)$, while the in-house method reported similar results as shown in figure 3 , samples tested Chisquare and fishers exact analysis showed no significant difference between results of both methods with a Pvalue $>0.999$. In addition all our eight negative SARS-COV2 samples were reported also negative by our FinHM assay. When the cycle threshold for any target gene crossed the 
threshold line within 40 cycles, the sample was considered diagnostically positive for SARS-CoV-2. All the $\mathrm{Ct}$ values from both platforms for all the 266 Patient samples that were obtained from SCDC and tested. The cycle threshold $(\mathrm{Ct})$ at which each RNA product was detected below $\mathrm{Ct}$ of 40 across all genes and ranged from $\mathrm{Ct}$ of 20.3 to $\mathrm{Ct}$ of 36.74 . These results indicate that the automated FinHM extraction was successful in extracting SARS-COV-2 viral RNA from patient samples and showed its effectiveness in the isolation and detection of SARS-COV-2 viral RNA.

\subsection{Automated FinHM Successfully Isolates SARS-COV-2 RNA Form Plasma spiked with the Virus Particles to low Titration and from Patient Plasma Samples}

To determine the capacity of FinHM to extract SARSCOV-2 from plasma we conducted a series of experiments using titrations of SARS-COV-2 whole virus propagated in the laboratory. Since the virus shedding in blood is almost $1 \%$, we started the dilution and titration from 500 copies down to $<10$ copies and spiked a control plasma from donor that are negative for SARS-COV-2 infection then subsequent extraction followed by RT-qPCR. The purpose of this experiment is to determine if our FinHM extraction platform is suitable for detecting SARS-COV-2 RNA in a plasma of COVID-19 positive patient, which are reported at low viral load and frequency. In this series of experiments the FinHM extraction was successful in SARS-COV-2 viral extraction, which was evident by the detection of the viral RNA after a Single-Step RT-qPCR using the Thermo Fisher assay (Table 4). All cycle thresholds for detection in this experiment were under Ct 40 and ranged from 19.19 to 34.46 . This finding indicates the promising usefulness for the FinHM platform to extract SARS-COV-2 RNA from patient blood samples. To test this possibility, we obtained 16 blood samples and extracted the viral RNA using FinHM platform and followed by ThermoFisher assay. This method detected SARS-COV-2 in 1 of the 16 samples with $\mathrm{Ct}$ values of 31.7 for $\mathrm{S}$ gene, 31.0 for ORF1b gene and 31.1 for $\mathrm{N}$ gene (Figure 4).

A)
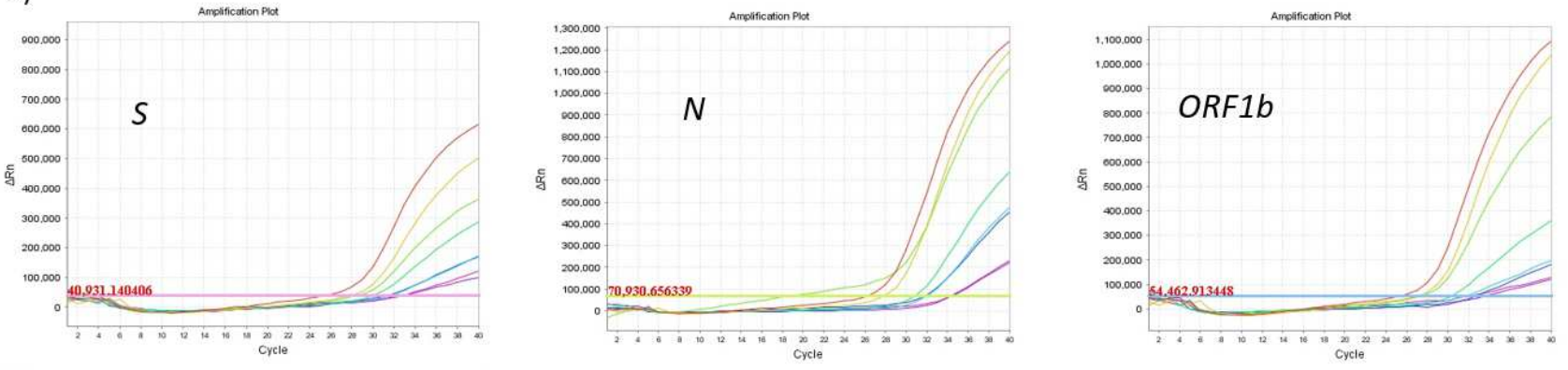

B)
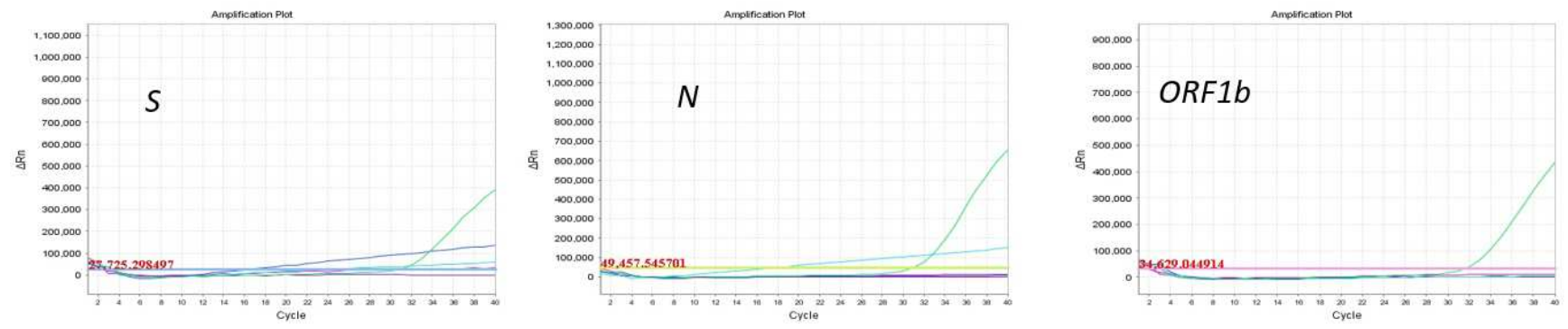

Figure 4. Detection of SARS-COV-2 virus in plasma using in-house assay after FinHM viral RNA extraction. SARS-COV-2 copy number titrations spiked into healthy donor plasma, top panel (A) and in COVID-19 patients bottom panel (B. Y axis is $\triangle R n$ and X axis is cycle thresh-hold and associated copy numbers and $C t$ values are seen in Table 4.

\subsection{In-House Multiplexing RT-qPCR Sensitivity and Limit of Detection}

To determine the in-House RT-qPCR sensitivity and its limit of detection we performed a standard curve experiment using SARS-COV-2 RNA template with one step titrations from 50,000 copies to 5 copies (Figure 5, and Table 5). In these experiments the In-House RT-qPCR was robust in detecting SARS-COV-2 RNA down to 0 copies and Pearson correlation showed a significant negative correlation between the $\mathrm{Ct}$ values and titrations (the higher the dilution the smaller the Ct value), with $-0.8,-0.8,-0.7$ for $E, \operatorname{RdRp} 2$, and RdRp4 respectively (Figure 6). 

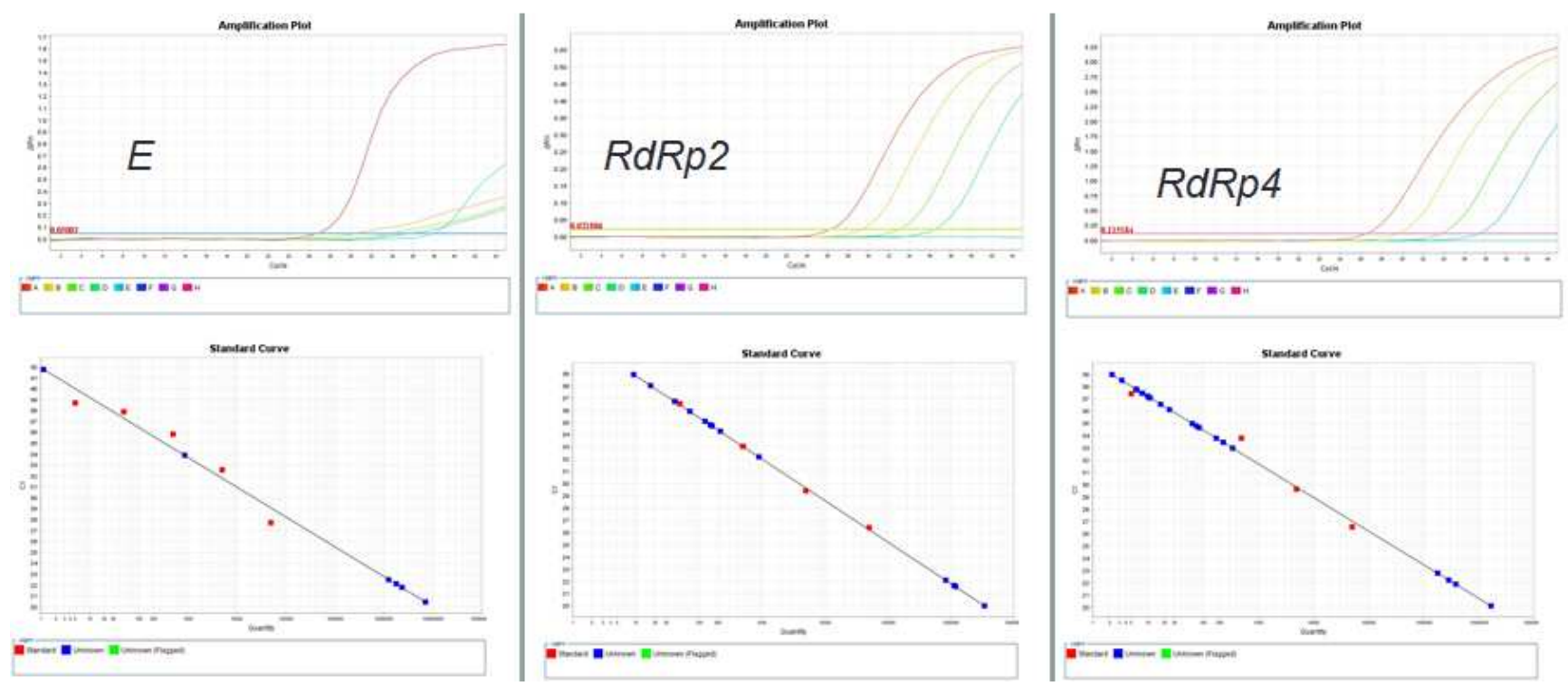

Figure 5. In-House Multiplexing RT-qPCR sensitivity analysis. Amplification curves upper panel and standard curves lower panel for E, RdRp2, RdRp4 and $N$ genes for RNA template at titration RNA template titrations $(50,000$ copies to $<10$ copies). Upper panel: $Y$ axis is $\Delta R n$ and $X$ axis is cycle thresh-hold and associated copy numbers and $C t$ values. Lower panel: $Y$ axis is $C t$ values and and $X$ axis copy number.

Table 4. The limit of detection of titrated SARS-COV-2 virus after FinHM automated viral RNA extraction process from plasma using ThermoFisher assay.

\begin{tabular}{llllll}
\hline SARS-COV-2 & \multicolumn{2}{l}{ Ct (ThermoFisher) } \\
\cline { 2 - 6 } (Copy number) & S & ORF1b & N & AVG & STDEV \\
\hline 500 & 24.94 & 25.70 & 26.44 & 25.69 & 0.61 \\
250 & 27.48 & 27.38 & 27.99 & 27.62 & 0.27 \\
125 & 28.17 & 27.71 & 29.99 & 28.62 & 0.98 \\
62.5 & 29.72 & 30.50 & 30.89 & 30.37 & 0.49 \\
31.25 & 31.20 & 31.83 & 32.10 & 31.71 & 0.38 \\
15.6 & 31.82 & 32.84 & 31.83 & 32.16 & 0.48 \\
7.8 & 32.10 & 33.68 & 34.48 & 33.42 & 0.99 \\
3.9 & 32.46 & 34.46 & 34.51 & 33.81 & 0.95 \\
0 & $* U D$ & UD & UD & 0 & 0 \\
\hline
\end{tabular}

*UD is Undetermined.

Table 5. SARS-COV-2 RNA templet standard prepared by titration from 50,000 to $<5$ viral copies with the corresponding CT value of our in-house PCR Detection of after FinHM extraction process.

\begin{tabular}{llll}
\hline Sample & Gene & Ct & Dilutions \\
\hline ST-1 & E gene & 27.71296 & 50000 \\
ST-2 & E gene & 32.57232 & 5000 \\
ST-3 & E gene & 35.852 & 500 \\
ST-4 & E gene & 37.91301 & 50 \\
ST-5 & E gene & 38.709 & 5 \\
ST-6 & E gene & Undetermined & 0 \\
ST-1 & RdRp2 & 26.40458 & 50000 \\
ST-2 & RdRp2 & 29.41998 & 5000 \\
ST-3 & RdRp2 & 33.06795 & 500 \\
ST-4 & RdRp2 & 36.52005 & 50 \\
ST-5 & RdRp2 & Undetermined & 5 \\
ST-6 & RdRp2 & Undetermined & 0 \\
ST-1 & RdRp4 & 26.54591 & 50000 \\
ST-2 & RdRp4 & 29.64758 & 5000 \\
ST-3 & RdRp4 & 33.80149 & 500 \\
ST-4 & RdRp4 & 35.90009 & 50 \\
ST-5 & RdRp4 & 37.44997 & 5 \\
ST-6 & RdRp4 & Undetermined & 0 \\
\hline
\end{tabular}

*UD is Undetermined.

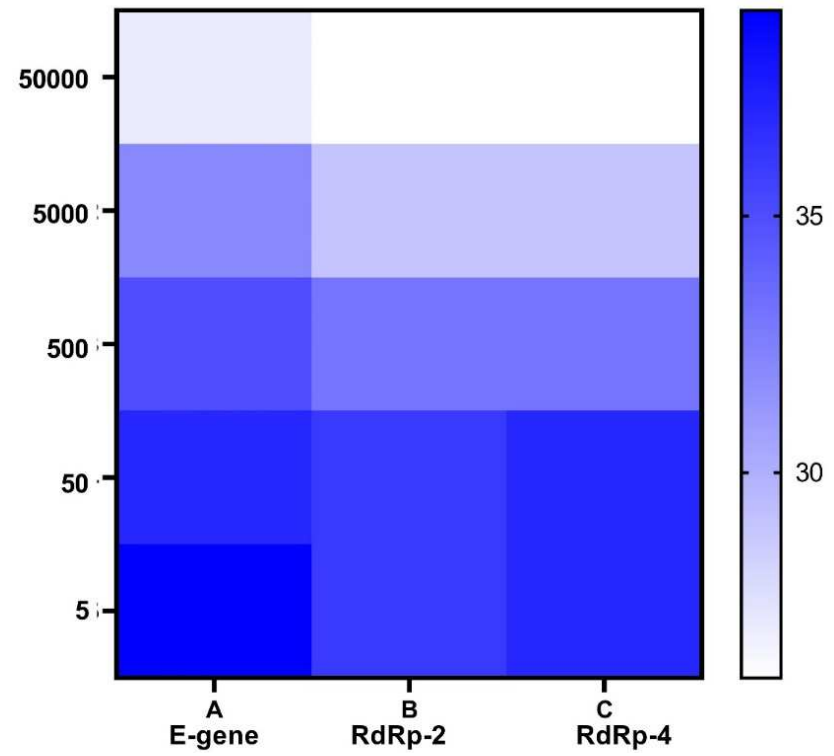

Figure 6. In-House Multiplexing RT-qPCR correlation between Ct values and titrations. Pearson correlation shows a significant negative correlation between the Ct values and titrations, the higher the dilution the smaller the Ct value with -0.8, -0.8, -0.7 for E, RdRp2, and RdRp4 genes respectively.

\section{Discussion}

On the 11th of March 2020, the WHO have declared the SARS-CoV-2 caused infection as pandemic. As of $21^{\text {st }}$ October global report shoes near Forty one million confirmed cases and over one Million deaths (https://www.who.int/docs/default-

source/coronaviruse/situation-reports/20200427-sitrep-98covid-19.pdf?sfvrsn=90323472_4). There are multiple reasons for this high rate of virus transmission, one of which is that significant number of cases are asymptomatic with capacity to spread the virus [8] in addition to the delayed measures that has been taken in response to this viral 
infection [9]. Accordingly, it has been important to perform mass testing to confirm and isolate positive subjects to counter global outbreak. This can be seen in some countries such as Germany and South Korea, in which the case to fatality rates dropped to less than $0.5 \%$ probably largely because of their mass testing efforts [10]. In addition, the mass testing in China played an important role in controling the spread of SARS-CoV-2 [11]. Yet, very few countries have used the mass testing approach for diagnostic SARSCOV -2 and this could be for many reasons, most importantly the limited resources for testing and budget restraints.

Although SARS-COV-2 extraction and RT-qPCR tests can be purchased, the huge world-wide demand in a short period of time created unprecedented shortage which hindered mass testing efforts. Thus, we developed a low-cost in-house RNA extraction solution suitable for viral RNA isolation, using commonly available and cost-effective reagents and chemicals and adapted a method that is suitable for automation and potentially for mass testing. We show in this report that this platform is robust and has been a successful to extract SARS-COV-2 RNA in clinical samples as detected by our in-house PCR assay. The platform that we have established has the capacity of extracting viral RNA from 192 samples per 1 hour per automation machine (Hamilton System), or 96 samples per 30 minutes per automation machine (KingFisher Flex system from Thermofisher), followed by 1.5 hours for RT-qPCR; a total of $\sim 3$ hours including preparations with a low cost of down to $\$ 0.80$ for the extraction and $\$ 3.80$ for the in-house PCR assay per sample. We have adapted the one-step real-time reverse transcriptase polymerase chain reaction RT-qPCR-based assays based on primer-probe assays (TaqMan) which is the gold standard for SARS-COV-2 diagnostics for upper and lower respiratory specimens. This assay utilizes oligonucleotide primers and labeled probes (TaqMan $\left.{ }^{\circledR}\right)$ to increase the sensitivity and specificity levels. The adapted set of primers and probes are among the list that have been evaluated and recommended by $\mathrm{CDC} / \mathrm{WHO}$ for any in house assay development for SARS-COV-2 testing. Despite considering this nucleic acid test as a gold-standard, several studies have reported false-negative results, and the reported sensitivity is up to $80 \%[2,12,13]$.

The analytical sensitivity and efficiency of SARS-COV-2 RT-qPCR primer-probe sets vary across different assays. At viral load of 500 copies and higher, the primer-probe sets have comparable sensitivities with $\mathrm{Ct}$ values ranging between 30 and 40 cycles, however at a lower copy number some probes have lower sensitivity than others [14]. Other factors may also affect detection of SARS-COV-2 in samples including sample collection time, site, storage and transport, hence most kit-based assays include 2 or 3 genes for SARSCOV-2 for testing. WHO, for instance recommends at least 3 target genes of E and RdRp2 and RdRp4 genes for SARSCOV-2 [4]. Two recent studies have evaluated the different sets of primers and probes recommended by CDC/WHO [14, 15], one reported that the RdRp and E are among the best to be tested with high specificity for SARS-CoV-2, with no cross-reactivity with other respiratory viruses and with a limit of detection of about 790 viral copies [14], while another report indicates that the lower sensitivities failed to reach 500 copies [15]. In this study we tested the limit of detection of SARS-COV-2 after automated RNA extraction by two different PCR assays, in which the commercially available assay and our in-house assay, both assay reported limit of detection of 46 copies for SARS-COV-2. In addition, the automated viral RNA extraction using this low-cost method followed by the In-House assay have detected SARS-COV-2 patient samples in capacity similar to that of the FDA approved ThermoFisher assay.

Moreover, we utilized the FinHM to extract SARS-COV-2 from blood samples. Since there are studies emerging that discusses the disease severity and its association with SARSCOV-2 viremia [15]. Establishing such platform will help the clinical treatment and disease management. We have received 16 blood samples from 16 different patients, our assay detected SARS-COV-2 in 1 out of 16 blood samples taken from patients admitted for treatment from SARS-COV2 infection, with a reported viral load of 8 SARS-COV-2 virus particles. Our future approach is to validate this on a larger scale of patients' samples.

The different reports on test sensitivity and viral load measurements of viral levels may reflect that no standardized process exists yet [7]. In addition, there is no established threshold for interpretation of viral loads, which may vary in different labs, assay used and the different targeted genes. In addition, the Detection rates in each sample type may vary from patient to patient and may change over the course of individual patients' illnesses [7]. The In-House assay can be utilized to be both qualitative and quantitative assay that enables the laboratory to measure viral load. Since the limit of detection is as low as 5 copies of RNA template with significant negative correlation between titration and $\mathrm{Ct}$ values $(50,000$ to 5 copies), this standard curve can be used to establish quantitative analysis to determine viral load in tested samples.

\section{Conclusions}

In conclusion, due to the urgent need for mass testing for SARS-COV-2, we report a low cost, reliable and sensitive viral RNA extraction automated platform for SARS-COV-2 and RT-qPCR. This method provides a promising alternative method given further clinical validation and FDA approval is obtained. In addition, this platform and protocol can be utilized in the future for any emergent or re-emergent pathogen as a preparedness program for any epidemic or pandemic thereat.

\section{List of Abbreviations}

Coronaviridae (CoVs), Acute Respiratory Syndrome Coronavirus (SARS-CoV), Middle East Respiratory Syndrome Coronavirus (MERS-CoV), Reverse transcription quantitative polymerase chain reaction (RT-qPCR), Centre 
for Disease Control (CDC), World Health Organization (WHO) Saudi Center for Disease control (SCDC), Full inhouse method (FinHM), Cycle threshold $(\mathrm{Ct})$ and NonTemplet control (NTC).

\section{Declarations}

All authors have given final approval of this manuscript to be published. In addition have agreed to be accountable for all aspects of the work in ensuring that questions related to the accuracy or integrity of any part of the work are appropriately investigated and resolved.

\section{Ethical Approval and Consideration}

The study was conducted according the Declaration of Helsinki. Ethical approval was obtained from the Office of Research Affairs at our institution and accordingly this study was approved by the institutional review board at King Faisal Specialist Hospital and Research Centre, Riyadh (KFSHRCRiyadh) IRB, (RAC Approval \# 2200031).

\section{Consent for Publication}

Not Applicable, this manuscript does not contain any Individual person's data in any form. The study was approved by the institutional research committee as deidentified samples that were left over after the completion of diagnostic tests; hence this study requires no consenting as per institutional ethics committee regulations.

\section{Availability of Data and Materials}

All data generated and analyzed during this study are included in this published article [and its supplementary information files].

\section{Competing Interest}

The authors declare that they have no competing interests.

\section{Funding}

Our institute; King Faisal Specialist Hospital \& Research Centre, Riyadh, Saudi Arabia as part of COVID-19 testing fund funded this study.

\section{Additional Data}

Supplementary table includes the RT-qPCR results for all the samples analyzed $(n=274)$ by both methods; the Thermofisher and the inhouse method results.

\section{Author Contributions}

M. A. M. ; methodology, M. A. M, K. A, R. B, L. M. I. A,
N. A, S. A, L. A, A. J, A. Z; software, A. J, A. Z X. X. ; validation, A. B, J. A, AA; formal analysis, M. AM, K. R, T. R. ; investigation, M. A. M, K. A; resources, M. AM, H. A; data curation, M. A. M, K. A; writing-original draft preparation, K. A; writing-review and editing, M. AM; visualization, M. A. M, K. A; supervision, MAM, project administration, LA. ; funding acquisition, MAM. All authors have read and agreed to the published version of the manuscript.

\section{Acknowledgements}

We acknowledged King Faisal Specialist Hospital and Research Centre (General Organization) for funding, resources and support. We acknowledged King Faisal Specialist Hospital and Research Centre (General Organization) for funding, resources and support. We would also want to thank Dr. Brian Meyer for his scientific discussion and feedback, Dr. Khalid Abu Khabar, and Dr. Ghazi Alsbeih for support and technical contribution, and Mr. Abdullah Alhadheq for technical expertise and modifying the automation script. We would also like to thank Dr. Sherif El-Kafrawy, Ahmed Hassan and Ahmed Tolah from the special infectious agent unit at King Fahd medical research center, King Abdulaziz University, Jeddah for his help in propagating and isolating SARS-COV-2 virus.

\section{References}

[1] Su S, Wong G, Shi W, Liu J, Lai ACK, Zhou J, et al. Epidemiology, Genetic Recombination, and Pathogenesis of Coronaviruses. Trends Microbiol. 2016; 24 (6): 490-502.

[2] Zhu N, Zhang D, Wang W, Li X, Yang B, Song J, et al. A Novel Coronavirus from Patients with Pneumonia in China, 2019. N Engl J Med. 2020; 382 (8): 727-33.

[3] Chu DKW, Pan Y, Cheng SMS, Hui KPY, Krishnan P, Liu Y, et al. Molecular Diagnosis of a Novel Coronavirus (2019nCoV) Causing an Outbreak of Pneumonia. Clin Chem. 2020; 66 (4): 549-55.

[4] Corman VM, Landt O, Kaiser M, Molenkamp R, Meijer A, Chu DK, et al. Detection of 2019 novel coronavirus (2019$\mathrm{nCoV}$ ) by real-time RT-PCR. Euro surveillance: bulletin Europeen sur les maladies transmissibles $=$ European communicable disease bulletin. 2020; 25 (3): 2000045.

[5] Cheng MP, Papenburg J, Desjardins M, Kanjilal S, Quach C, Libman M, et al. Diagnostic Testing for Severe Acute Respiratory Syndrome-Related Coronavirus-2: A Narrative Review. Annals of internal medicine. 2020: M20-1301.

[6] Wells CR, Sah P, Moghadas SM, Pandey A, Shoukat A, Wang $\mathrm{Y}$, et al. Impact of international travel and border control measures on the global spread of the novel 2019 coronavirus outbreak. Proceedings of the National Academy of Sciences of the United States of America. 2020; 117 (13): 7504-9.

[7] Pasteur I. COVID-19 Disease (Novel Coronavirus): Institute Pasteur 2020 [Available from: https: //www.pasteur.fr/en/medical-center/disease-sheets/covid-19disease-novel-coronavirus. 
[8] Bai Y, Yao L, Wei T, Tian F, Jin D-Y, Chen L, et al. Presumed Asymptomatic Carrier Transmission of COVID-19. JAMA. 2020; 323 (14): 1406-7.

[9] Dong E, Du H, Gardner L. An interactive web-based dashboard to track COVID-19 in real time. Lancet Infect Dis. 2020; 20 (5): 533-4.

[10] Liu R, Han H, Liu F, Lv Z, Wu K, Liu Y, et al. Positive rate of RT-PCR detection of SARS-CoV-2 infection in 4880 cases from one hospital in Wuhan, China, from Jan to Feb 2020. Clin Chim Acta. 2020; 505: 172-5.

[11] Huang C, Wang Y, Li X, Ren L, Zhao J, Hu Y, et al. Clinical features of patients infected with 2019 novel coronavirus in Wuhan, China. Lancet. 2020; 395 (10223): 497-506.

[12] Nalla AK, Casto AM, Huang MW, Perchetti GA, Sampoleo R, Shrestha L, et al. Comparative Performance of SARS-CoV-2
Detection Assays using Seven Different Primer/Probe Sets and One Assay Kit. J Clin Microbiol. 2020.

[13] Vogels CBF, Brito AF, Wyllie AL, Fauver JR, Ott IM, Kalinich $\mathrm{CC}$, et al. Analytical sensitivity and efficiency comparisons of SARS-COV-2 qRT-PCR primer-probe sets. medRxiv. 2020: 2020.03.30.20048108.

[14] Young BE, Ong SWX, Kalimuddin S, Low JG, Tan SY, Loh J, et al. Epidemiologic Features and Clinical Course of Patients Infected With SARS-CoV-2 in Singapore. Jama. 2020; 323 (15): 1488-94.

[15] Patel R, Babady E, Theel ES, Storch GA, Pinsky BA, St George K, et al. Report from the American Society for Microbiology COVID-19 International Summit, 23 March 2020: Value of Diagnostic Testing for SARS-CoV-2/COVID19. mBio. 2020; 11 (2). 